\title{
Effect of Living Environment Factors on Quality of Life in Patients With Chronic Thromboembolic Pulmonary Hypertension After Completion of Balloon Pulmonary Angioplasty

\author{
Yosuke Morimoto, PhD; Satoshi Ikeda, MD, PhD; Yuki Yamagata, MD, PhD; \\ Ryo Kozu, PhD; Hiroaki Kawano, MD, PhD; Koji Maemura, MD, PhD
}

\begin{abstract}
Background: This study investigated factors related to quality of life (QoL) in patients with chronic thromboembolic pulmonary hypertension who completed balloon pulmonary angioplasty (BPA).

Methods and Results: Patient QoL and living environment after BPA were evaluated prospectively using the 5-level EQ-5D questionnaire and International Physical Activity Questionnaire Environmental Module (IPAQ-E), respectively. Patients were mailed copies of both surveys. In addition, we reviewed patient charts and collected retrospective clinical data. Relationship between the clinical data and QoL and environmental living factors were investigated. Of the 33 subjects mailed the surveys, sufficient responses were obtained from $22(71 \%)$. Spearman's rank correlation coefficient showed that psychiatric disorders $(r=-0.6865, P<0.01)$ and IPAQ-E Question $5(r=0.5192, P=0.02)$, Question $6(r=0.5265, P=0.02)$, and Question $13(r=0.4552, P=0.04)$ were significantly correlated with $\mathrm{EQ}-5 \mathrm{D}$ scores after $\mathrm{BPA}$.
\end{abstract}

Conclusions: A living environment that was difficult to walk around was associated with a worse QoL. A multidisciplinary approach will be required to improve QoL even after completion of BPA treatment.

Key Words: Balloon pulmonary angioplasty; Environment; Pulmonary hypertension; Quality of life

C hronic thromboembolic pulmonary hypertension (CTEPH) is a rare disease and a form of pulmonary hypertension caused by chronic obstruction of the pulmonary artery due to organized thrombi, leading to right heart failure. Dyspnea on exertion is clinically common in CTEPH and reduces quality of life (QoL). ${ }^{1}$ According to the CTEPH treatment algorithm, pulmonary endarterectomy (PEA) is considered the only curative treatment for operable CTEPH patients. ${ }^{2}$ Although PEA is an established surgical therapy for CTEPH, PEA is limited to central-type CTEPH and is not feasible for peripheral-type CTEPH. In CTEPH cases that are not eligible for surgery, balloon pulmonary angioplasty (BPA) has been performed as an effective therapy for peripheral-type CTEPH and residual pulmonary hypertension after PEA, yielding outcomes comparable to those of surgical treatment. 3,4

BPA is an interventional treatment that uses a balloon catheter to dilate thromboembolic pulmonary arteries and is less invasive than the surgical procedure PEA. Darocha et al reported a significant improvement in QoL after a series of BPAs, suggesting that QoL improvement may be an additional aim of BPA treatment. ${ }^{5}$ QoL is not only dependent on pulmonary hemodynamics because "QoL" is an overarching term for the quality of various domains in life. The living environment is also related to QoL. Egoshi et al reported that an environment that makes going out difficult was related to a low QoL in chronic obstructive pulmonary disease patients with dyspnea, a common symptom of CTEPH. ${ }^{6}$ Although BPA can

Received November 30, 2020; revised manuscript received February 28, 2021; accepted March 5, 2021; J-STAGE Advance Publication released online April 3, 2021 Time for primary review: 27 days

Department of Physical Therapy, Faculty of Rehabilitation, Kobe Gakuin University, Kobe (Y.M.); Department of Rehabilitation Medicine, Nagasaki University Hospital, Nagasaki (Y.M., R.K.); Department of Cardiovascular Medicine (S.I., H.K., K.M.), Department of Cardiopulmonary Rehabilitation Science (R.K.), Nagasaki University Graduate School of Biomedical Sciences, Nagasaki; and Department of Cardiovascular Medicine, Nagasaki Prefecture Shimabara Hospital, Nagasaki (Y.Y.), Japan

K.M. is a member of Circulation Reports' Editorial Team.

Mailing address: Yosuke Morimoto, PT, PhD, Department of Physical Therapy, Faculty of Rehabilitation, Kobe Gakuin University, 518 Arise, Ikawadanicho, Nishi-ku, Kobe 651-2180, Japan. E-mail: morimoto@reha.kobegakuin.ac.jp

All rights are reserved to the Japanese Circulation Society. For permissions, please e-mail: cr@j-circ.or.jp

ISSN-2434-0790 


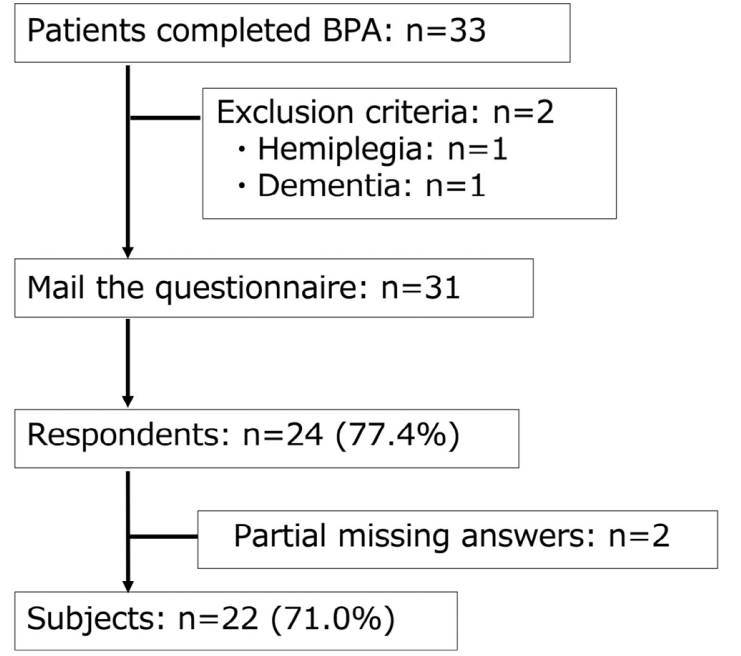

Figure 1. Study flow chart. Questionnaires were sent to 31 patients: 24 patients responded but 2 did not complete the questionnaire, leaving 22 patients included in the present study. BPA, balloon pulmonary angioplasty.

improve pulmonary hypertension and QoL, it has not been fully elucidated what factors affect QoL in CTEPH patients who have undergone BPA. The aim of the present study was to investigate the factors related to QoL in patients who underwent BPA.

\section{Methods}

A prospective study was conducted in Japanese patients diagnosed with CTEPH and who had undergone BPA at Nagasaki University Hospital between September 2013 and October 2018. Patients who were not independent in activities of daily living or those who had dementia, making it difficult to fill out a self-report questionnaire, were excluded. A self-report questionnaire was mailed to patients' homes, and clinical data were collected from their medical records. Findings of pulmonary hemodynamics and arterial blood gas assays during right heart catheterization and data from blood tests, such as N-terminal pro B-type natriuretic peptide (NT-proBNP) and C-reactive protein (CRP), on admission for BPA or cardiac catheterization were analyzed in this study.

We used revised definitions for each comorbidity as follows. Hypertension was defined as either receiving treatment with antihypertensive agents or having a systolic blood pressure of $\geq 140 \mathrm{mmHg}$ and/or a diastolic blood pressure of $\geq 90 \mathrm{mmHg}^{7}$ Valvular heart disease was defined as moderate to severe according to standard echocardiographic criteria. ${ }^{\mathbf{8}}$ Diabetes was defined as fasting blood glucose $\geq 126 \mathrm{mg} / \mathrm{dL}, \mathrm{HbA} 1 \mathrm{c} \geq 6.5 \%$, or taking antidiabetic drugs. ${ }^{9}$ Deep vein thrombosis (DVT) was diagnosed on the basis of results of medical examinations, D-dimer measurement, and imaging findings of lower extremity veins. ${ }^{10}$ Sleep apnea syndrome (SAS) was defined by polysomnography as an apnea-hypopnea index $\geq 5 .{ }^{11}$ Patients were considered to have a psychiatric disorder if they had been diagnosed by psychiatrists or had taken antipsychotic agents. $^{12}$
This study was approved by the Human Ethics Review Committee of Nagasaki University Hospital (Reference no. 19052002), and all patients provided written informed consent. This study was conducted in accordance with the Declaration of Helsinki.

\section{Assessment of QoL and Living Environment}

We prospectively evaluated QoL and the living environment using self-reported questionnaires $>6$ months after the final BPA session. QoL was evaluated using the 5-level EQ-5D questionnaire. The EQ-5D was developed as an instrument that is not specific to disease but is standardized and can be used as a complement to existing health-related QoL measures. ${ }^{13}$ The EQ-5D is an indirect method of measuring utility and contains questions in 5 domains: mobility, self-care, usual activities, pain/discomfort, and anxiety/ depression. These questions were answered using a 5-point categorical rating scale, varying from 'no problems at all' to 'extreme problems (unable to do)'. These values were expressed in numbers, ranging from 0 (dead) to 1 (full health). A Japanese version of the EQ-5D developed by Tsuchiya et al was used in the present study. ${ }^{\mathbf{1 4}}$

The attributes of the living environment were evaluated using the International Physical Activity Questionnaire Environmental Module (IPAQ-E). The IPAQ-E contains 17 questions regarding: residential density, access to shops, access to public transport, presence of sidewalks, presence of bike lanes, access to recreational facilities, crime at night, traffic safety when walking, social environment, aesthetics, household motor vehicles, 4-way intersections, well-maintained sidewalks, well-maintained places for cycling, traffic safety when cycling, crime during the day, and access to public facilities. These items refer to the surrounding environment that a person can reach by walking $10-15 \mathrm{~min}$ from his or her residence. Fifteen of the 17 items (i.e., all except residential density and household motor vehicles) included statements explaining neighborhood features related to physical activity, with possible responses being "strongly disagree", "somewhat disagree", "somewhat agree", and "strongly agree". Responses for these environmental variables were classified into 2 categories: agreement ("strongly agree" and "somewhat agree") or disagreement ("somewhat disagree" and "strongly disagree"). ${ }^{15}$ In terms of residential density, the choice of "detached single-family residences" formed a category indicating low residential density, whereas other forms of residence were included in a category indicating high residential density. Responses to the number of household motor vehicles were categorized as "none" and " $\geq 1$ ". The translation process, description of each item, and reliability of this scale have been reported elsewhere. ${ }^{\mathbf{1 6}}$

\section{Statistical Analysis}

Spearman's rank correlation coefficient was used to identify clinical variables associated with EQ-5D scores. For the analyses, responses to 17 environmental variables were converted into dichotomous variables using the method used in previous studies. ${ }^{15}$ Candidate factors were selected according to general factors related to CTEPH, as well as to social information and living environment that may be related to QoL, and that contributed significantly to the outcome of Spearman's rank correlation coefficient $(\mathrm{P}<0.05)$. The Wilcoxon signed-rank test was used to examine changes in findings of cardiac catheterization, blood test, and 6-min walk distance (6MWD) before and 
after completion of BPA treatment. Changes in parameters before and after BPA $(\Delta)$ were calculated by subtracting baseline values from values after BPA treatment.

All analyses were performed using the JMP ${ }^{\circledR}$ Pro 13 Version 13.0.0 (SAS Institute, Cary, NC, USA). Data are expressed as the median with interquartile range (IQR) or as percentages, as appropriate. The level of significance was set at 2-tailed $\mathrm{P}<0.05$.

\section{Results}

Questionnaires were sent to 31 patients, excluding the 2 patients who met the exclusion criteria, and received answers from 24 patients $(77.4 \%)$. Two patients did not completely fill out the questionnaire, so 22 patients $(71.0 \%)$ were finally included in the study (Figure 1).

\section{Baseline Characteristics}

The baseline characteristics of the patients are presented in Table 1. The number of patients in World Health Organization (WHO) functional classes I, II, III, and IV was $0,7(31.8 \%), 15(68.2 \%)$, and 0 , respectively. The median mean pulmonary arterial pressure (mPAP) was $40.5 \mathrm{mmHg}$ and the maximum mPAP was $56 \mathrm{mmHg}$. The median NT-proBNP concentration was $312.2 \mathrm{pg} / \mathrm{mL}$, and concentrations $<400 \mathrm{pg} / \mathrm{mL}$, indicating heart failure, were found in 9 individuals (42.9\%). Among the comorbidities, hypertension was the most common $(40.9 \%)$, followed by psychiatric disorders $(18.2 \%)$. Patients with a psychiatric disorder were taking sulpiride, zotepine, and/or blonanserin. The median 6MWD, an indicator of exercise tolerance, was $319.5 \mathrm{~m}$. The median number of BPA sessions was 5 , and at least 3 BPA sessions were performed for each patient.

\section{Changes in Pulmonary Hemodynamics, Arterial Oxygenation, NT-proBNP, and 6MWD Following BPA}

Changes in parameters before and after completion of BPA treatment are shown in Figure 2. BPA significantly reduced $\mathrm{mPAP}$, pulmonary vascular resistance (PVR), and NT-proBNP concentrations and increased stroke volume (SV), partial pressure of arterial oxygen $\left(\mathrm{PaO}_{2}\right)$, and 6MWD. However, there was no significant change in cardiac output $(\mathrm{CO})$. After completion of BPA treatment, mPAP in 19 patients was $<20 \mathrm{mmHg}$. In addition, there was a median increase in 6MWD of $70 \mathrm{~m}$ (IQR 19.8$98.3 \mathrm{~m}$ ), from $319.5 \mathrm{~m}$ (IQR $241.5-421.5 \mathrm{~m}$ ) before BPA to $420.0 \mathrm{~m}$ (IQR 346.8-484.5 m) after BPA.

\section{EQ-5D Scores After BPA}

The median EQ-5D score was 0.825 , but had a wide distribution (0.389-1.000; Figure 3). More than half the patients responded with 'no problems at all' to items of self-care, pain/discomfort, and anxiety/depression. In items of activity, $43 \%$ of patients reported 'no problems at all' and 'slight problems'. In terms of mobility, the proportion of answers on the 5-point scale from 'no problems at all' to 'extreme problems (unable to do)' was 33\%, 43\%, 24\%, $0 \%$, and $0 \%$, respectively, with the variation in answers to this item being the largest among the 5 items on the EQ-5D.

\section{Relationship Between EQ-5D Scores and Patient Demographics, Changes in Pulmonary Hemodynamics, and IPAQ-E Scores}

Factors associated with the EQ-5D score, as assessed by

\begin{tabular}{|lc|}
\hline Table 1. Patient Characteristics & \\
Age (years) & $69.5[59.0-74.8]$ \\
Female sex & $17(77.7)$ \\
Height (cm) & $154.1[151.2-164.3]$ \\
Weight (kg) & $58.4[51.5-66.2]$ \\
BMI (kg/m²) & $23.83[22.20-26.04]$ \\
WHO FC I/II/II & $0(0) / 7(31.8) / 15(68.2)$ \\
No. BPA sessions & $5[4-6]$ \\
Comorbidity & \\
Hypertension & $9(40.9)$ \\
Valvular heart diseases & $2(9.1)$ \\
Diabetes & $2(9.1)$ \\
DVT & $2(9.1)$ \\
SAS & $1(4.5)$ \\
Psychiatric disorders & $4(18.2)$ \\
Social information & \\
Living alone & $5(22.7)$ \\
Employed & $5(22.7)$ \\
Using care insurance & $17(77.3)$ \\
Blood findings & \\
CRP (mg/dL) & $87.2[85.4-88.6]$ \\
Albumin (g/dL) & $6.08[0.04-0.25]$ \\
NT-proBNP (pg/mL) & $4.05[3.6-4.4]$ \\
PaO2 (Torr) & $312.2[101.4-758.7]$ \\
Right heart catheterization & $59.5[56.9-67.5]$ \\
Mean PAP (mmHg) & \\
Mean PAWP (mmHg) & $40.5[31.0-44.3]$ \\
PVR (wood unit) & $6.5[5.0-7.25]$ \\
Mean RAP (mmHg) & $8.2[5.9-9.6]$ \\
CO (L/min) & $4.0[2.8-6.0]$ \\
Cl (L/min/m²) & $4.2[3.6-4.8]$ \\
SV (mL) & $2.6[2.4-3.2]$ \\
6MWD (m) & $56.8[50.3-73.4]$ \\
HOT & $319.5[241.5-421.5]$ \\
SpO2 (\%) & $6(27.3)$ \\
\hline Valles arepresentedas the & \\
\hline
\end{tabular}

Values are presented as the median [interquartile range] or $n(\%)$. 6MWD, 6-min walk distance; BMI, body mass index; BPA, balloon pulmonary angioplasty; $\mathrm{Cl}$, cardiac index; $\mathrm{CO}$, cardiac output; CRP, C-reactive protein; DVT, deep vein thrombosis; HOT, home oxygen therapy; NT-proBNP, N-terminal pro B-type natriuretic peptide; $\mathrm{PaO}_{2}$, partial pressure of oxygen in arterial blood; PAP, pulmonary arterial pressure; PAWP, pulmonary arterial wedge pressure; PVR, pulmonary vascular resistance; RAP, right atrial pressure; SAS, sleep apnea syndrome; $\mathrm{SpO}_{2}$, saturation of percutaneous oxygen; SV, stroke volume; WHO FC, World Health Organization functional class.

single linear regression, are presented in Tables $\mathbf{2 - 4}$. Among patient demographics, an underlying psychiatric disorder was significantly associated with EQ-5D scores $(\beta=-0.19, \mathrm{P}<0.01)$. In addition, $\triangle \mathrm{PVR}(\mathrm{r}=0.5266, \mathrm{P}=0.01)$ and $\Delta \mathrm{SV}(\mathrm{r}=-0.5409, \mathrm{P}=0.01)$ were significantly associated with EQ-5D scores. Among the IPAQ-E items, questionnaire items 5 (the presence of bike lanes; $\mathrm{r}=0.5192, \mathrm{P}=0.02$ ), 6 (access to recreational facilities; $\mathrm{r}=0.5265, \mathrm{P}=0.02$ ), and 13 (a well-maintained sidewalk; $r=0.4552, \mathrm{P}=0.04$ ) were significantly associated with EQ-5D scores.

\section{Discussion}

The main findings of the present study are as follows: (1) 

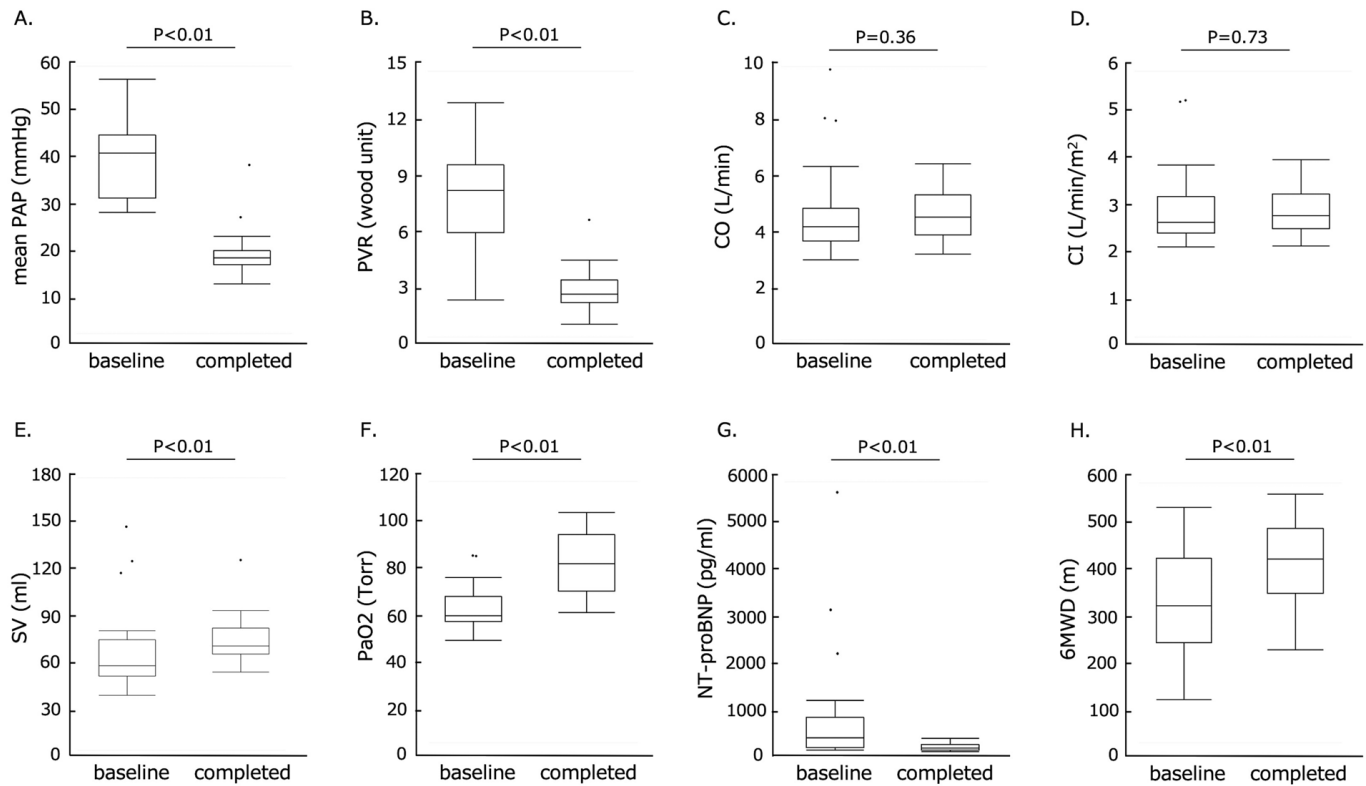

Figure 2. Changes in parameters before and after balloon pulmonary angioplasty. 6MWD, 6-min walk distance; $\mathrm{Cl}$, cardiac index; $\mathrm{CO}$, cardiac output; NT-proBNP, N-terminal pro B-type natriuretic peptide; $\mathrm{PaO}_{2}$, partial pressure of oxygen in arterial blood; PAP, pulmonary arterial pressure; PVR, pulmonary vascular resistance; SV, stroke volume.

A.

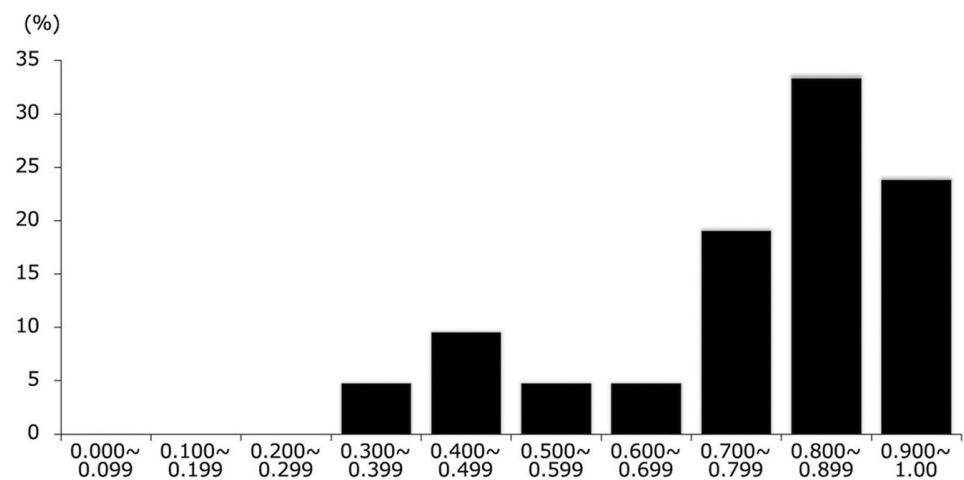

B.
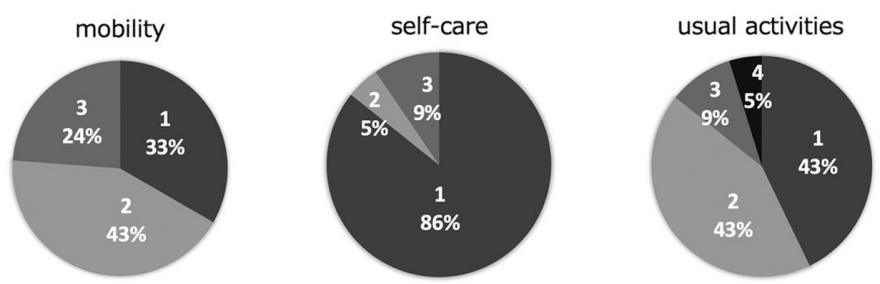

pain/discomfort
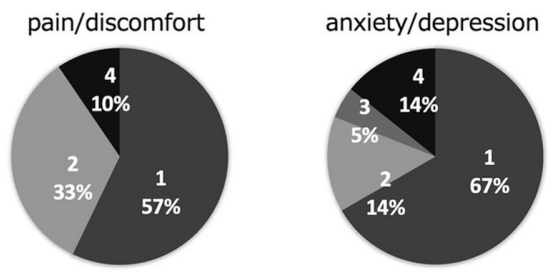

$1=$ no problems at all

$2=$ slight problems

$3=$ moderate problems

$4=$ severe problems

$5=$ extreme problems (unable to do)

Figure 3. (A) Distribution of 5-level EQ-5D scores for all items and (B) the proportion of the scores for each item. 


\begin{tabular}{|lcc|}
\hline \multicolumn{2}{|c|}{ Table 2. Correlations Between Patient Demographics and } \\
EQ-5D Scores & r & P value \\
Age, per year & 0.2222 & 0.33 \\
Female sex & 0.1862 & 0.42 \\
Height, per $1 \mathrm{~cm}$ & 0.3831 & 0.09 \\
Weight, per $1 \mathrm{~kg}$ & 0.2087 & 0.36 \\
BMI, per $1 \mathrm{~kg} / \mathrm{m}^{2}$ & 0.1094 & 0.64 \\
Comorbidity & & \\
Hypertension & 0.1469 & 0.53 \\
Valvular heart diseases & -0.0270 & 0.91 \\
Diabetes & 0.2978 & 0.19 \\
DVT & -0.0135 & 0.95 \\
SAS & -0.2606 & 0.25 \\
Psychiatric disorders & -0.6865 & $<0.01$ \\
BPA, per 1 session & -0.1219 & 0.60 \\
Living alone & 0.0931 & 0.69 \\
Employed & 0.3144 & 0.20 \\
Using care insurance & -0.3723 & 0.10 \\
\hline
\end{tabular}

Abbreviations as in Table 1.

\begin{tabular}{|c|c|c|}
\hline \multicolumn{3}{|l|}{ Blood tests } \\
\hline$\Delta \mathrm{NT}$-proBNP, per $100 \mathrm{pg} / \mathrm{mL}$ & 0.3555 & 0.11 \\
\hline$\Delta \mathrm{PaO}_{2}$, per 10 Torr & -0.2149 & 0.35 \\
\hline \multicolumn{3}{|l|}{ Right heart catheterization } \\
\hline$\Delta$ Mean PAP, per $1 \mathrm{mmHg}$ & 0.4159 & 0.06 \\
\hline$\Delta$ Mean PAWP, per $1 \mathrm{mmHg}$ & -0.0578 & 0.80 \\
\hline$\Delta \mathrm{PVR}$, per 1 wood unit & 0.5266 & 0.01 \\
\hline$\Delta \mathrm{CO}$, per $1 \mathrm{~L} / \mathrm{min}$ & -0.4168 & 0.06 \\
\hline$\Delta \mathrm{Cl}$, per $1 \mathrm{~L} / \mathrm{min} / \mathrm{m}^{2}$ & -0.4007 & 0.07 \\
\hline$\Delta S V$, per $1 \mathrm{~mL}$ & -0.5409 & 0.01 \\
\hline \multicolumn{3}{|l|}{ Exercise capacity } \\
\hline$\Delta 6 \mathrm{MWD}$, per $100 \mathrm{~m}$ & -0.2325 & 0.31 \\
\hline
\end{tabular}

Delta indicates the amount of change (completed BPA-baseline). Abbreviations as in Table 1.

BPA is an effective treatment for CTEPH because the parameters related to pulmonary hemodynamics and heart failure were improved following BPA treatment; (2) a greater improvement in these parameters was associated with a better QoL; (3) psychiatric disorders significantly reduced QoL; and (4) in terms of the living environment, factors that made it difficult for patients to go out reduced QoL. These findings suggest that various factors, such as hemodynamic changes and living environment, may affect the QoL of CTEPH patients who have completed BPA treatment.

The prognosis of medically treated CTEPH patients with mPAP $>30 \mathrm{mmHg}$ has been reported to be very poor. ${ }^{17}$ Indeed, inoperable CTEPH had a poor prognosis with historical therapy, with a 5-year survival rate of approximately $60 \%$. BPA is a promising treatment for peripheral-type CTEPH and inoperable CTEPH patients, with a better prognosis regardless of age. ${ }^{12,18}$ Furthermore,

\begin{tabular}{|lrc|}
\hline $\begin{array}{l}\text { Table 4. Correlations Between IPAQ-E Items and EQ-5D } \\
\text { Scores }\end{array}$ & \multicolumn{1}{c|}{$\mathbf{r}$} & P value \\
IPAQ-E item & -0.2337 & 0.31 \\
1. Residential density (yes) & 0.0336 & 0.89 \\
2. Access to shops (yes) & 0.3004 & 0.19 \\
3. Access to public transport (yes) & 0.1023 & 0.67 \\
4. Presence of sidewalks (yes) & 0.5192 & 0.02 \\
5. Presence of bike lanes (yes) & 0.5265 & 0.02 \\
6. Access to recreational facilities (yes) & -0.2273 & 0.32 \\
7. Crime at night (yes) & -0.3575 & 0.11 \\
8. Traffic safety when walking (yes) & 0.1323 & 0.57 \\
9. Social environment (yes) & 0.1345 & 0.56 \\
10. Aesthetics (yes) & 0.4204 & 0.06 \\
11. Household motor vehicles (yes) & -0.0696 & 0.76 \\
12. 4-Way intersections (yes) & 0.4552 & 0.04 \\
13. Well-maintained sidewalks (yes) & 0.0651 & 0.78 \\
14. Well-maintained places for cycling (yes) & 0.0713 & 0.76 \\
15. Traffic safety when cycling (yes) & -0.0724 & 0.76 \\
\hline 16. Crime during the day (yes) & 0.4277 & 0.05 \\
\hline 17. Access to public facilities (yes) &
\end{tabular}

IPAQ-E, International Physical Activity Questionnaire Environmental Module.

several reports have shown that BPA improves QoL. Darocha et al reported that baseline QoL in patients with CTEPH was significantly worse than in the healthy population and, after a series of BPA procedures, QoL improved significantly on all scales, as well as on scales normalized relative to the healthy population, except for pain experience. ${ }^{5}$ Previously, the effects of treatment on QoL were evaluated in CTEPH patients who had undergone PEA and/or had been treated pharmacologically with specific drugs. Therefore, the report of Darocha et al showed, for the first time, that BPA is an effective treatment that significantly improves QoL. In 2020, 2 studies reported the effects of BPA on QoL in patients with CTEPH. ${ }^{19,20}$ These studies confirmed that BPA improved pulmonary hemodynamics (mPAP and PVR), as well as QoL. Yoshimi et al reported that in patients with a significant hemodynamic improvement after PEA (a decrease in PVR $>50 \%$ compared with baseline), significant improvements were also seen QoL (using the 36-Item Short Form Health Survey [SF-36] questionnaire) within the domains of physical function, role-physical, general health, social functioning, role-emotional, and mental health, but that no improvements in QoL were found in patients in whom PVR decreased $<50 \%$ after PEA. ${ }^{21}$ A lower PVR was associated with a better QoL in a recent multivariate analysis of 128 CTEPH patients treated with PEA or medical therapy. ${ }^{22} \mathrm{~A}$ decline in PVR is an important factor in reducing dyspnea, thus making a patient's life more comfortable. These results are in agreement with those of the present study showing that a greater decrease in PVR following BPA was associated with a greater improvement in QoL. Thus, BPA may improve QoL by improving not only pulmonary hemodynamics, but also various pathological conditions. In addition, larger changes in BPA were associated with a better QoL.

In the present study, exercise capacity in all subjects improved after BPA compared with values before BPA; the median $\triangle 6 \mathrm{MWD}$ was $70-420 \mathrm{~m}$. However, no signifi- 
cant correlation between the $\triangle 6 \mathrm{MWD}$ and EQ-5D scores was found. Some studies also failed to find a significant correlation between the physical-related score of the SF-36 after completion of BPA treatment and improvements in the 6MWD, and in some cases an improvement in EQ-5D scores was not seen despite improvements in 6MWD following BPA.5,20 Fukui et al reported that 12 weeks of cardiac rehabilitation for patients with CTEPH who completed BPA effectively improves exercise capacity and QoL, but the effect of cardiac rehabilitation was limited to mental health. As such, 6MWD is thought to have little direct effect on QoL. ${ }^{23}$

It has been reported that patients taking oral antipsychotic medication are more likely to develop venous thromboembolism. ${ }^{\mathbf{2 4 , 2 5}}$ Furthermore, patients with chronic heart failure have been reported to have a significantly worse prognosis if they have concomitant depression. ${ }^{26}$ In the present study, $18.2 \%$ of subjects had psychiatric disorders, and underlying psychiatric disorders were related to a significant decrease in QoL. Saarni et al reported that schizoaffective disorder was associated with the largest decrease in QoL. ${ }^{27}$ Current depressive symptoms accounted for most of the effect, and the symptoms were the strongest predictors of poor QoL in psychotic disorders. Although pulmonary hemodynamics and heart failure were improved by BPA, it is difficult to improve the QoL of patients with psychiatric disorders. However, Tajima et al reported that there was no significant decrease in survival rate according to complications of psychiatric disorders in non-surgical CTEPH patients treated with pulmonary vasodilators. ${ }^{28}$ However, it should be noted that the aim of BPA in CTEPH patients with psychiatric disorders is to improve survival rather than QoL.

Regarding the association between QoL and the living environment, significant factors in the IPAQ-E were items 5 (the presence of bike lanes), 6 (access to recreational facilities), and 13 (well-maintained sidewalks). It has been reported that the presence of bike lanes and access to recreational facilities are significant correlates of daily walking activity. ${ }^{\mathbf{1 6}}$ It seems that these environmental factors are associated with ease of going out. Based on our experience in routine clinical practice, it is unlikely that exercise capacity after the rehabilitation program offered to our patients would restrict them from going out. Some patients needed home oxygen therapy before and during BPA treatment, and patients often do not like going outside if they experience dyspnea during exercise and do not want their neighbors to see them wearing a nose cannula. Patients' old habits may also reduce physical activity after BPA. A greater overall activity level was related to greater happiness, better function, and reduced mortality. ${ }^{29}$ For the "mobility" and "usual activities" domains in the EQ-5D, only a few patients answered "no problems at all" in this study. This suggests that exercise capacity has improved, but patients may not be able to make use of this improvement because of their living environment. By ensuring that patients have family support and/or by reviewing care services, QoL following BPA may be further improved if factors of the living environment related to QoL are no longer major barriers.

\section{Study Limitations}

The sample size in this study was small because CTEPH is a rare disease. Because of the small sample size, multiple analyses were not performed. The results should be inter- preted with caution because confounding factors may be present, and we could not establish independent factors. We believe that a multicenter investigation with a large sample size is necessary. We did not evaluate QoL using the EQ-5D before BPA, therefore changes in the QoL are unknown. Most patients in the present study were women $(77.7 \%)$. We do not think this is indicative of selection bias, because female patients outnumber male patients $(2.6: 1)$ in Japan. ${ }^{30}$

\section{Conclusions}

BPA is an effective treatment for improving pulmonary hemodynamics and exercise capacity in patients with CTEPH. In the present study we showed that a living environment that prevented patients from leaving the house was associated with lower QoL after BPA, indicating that there is still room for improvement in QoL in CTEPH patients who complete BPA treatment. Therefore, a continuous and multidisciplinary approach will be required to improve QoL in patients with CTEPH.

\section{Acknowledgments}

The authors thank the members of the Departments of Rehabilitation and Cardiovascular Medicine, Clinical Research Center, Nagasaki University Hospital and the Department of Cardiopulmonary Rehabilitation Science, Nagasaki University Graduate School of Biomedical Sciences for their support. This research would not have been possible without their leadership and cooperation.

\section{Sources of Funding}

This research did not receive any grant from any funding agency in the public, commercial, or not-for-profit sectors.

\section{Disclosures}

K.M. is a member of Circulation Reports' Editorial Team. The remaining authors have no conflicts of interest to declare.

\section{IRB Information}

This study was approved by the Human Ethics Review Committee of Nagasaki University Hospital (Reference no. 19052002).

\section{References}

1. Pfeuffer E, Krannich H, Halank M, Wilkens H, Kolb P, Jany B, et al. Anxiety, depression, and health-related QOL in patients diagnosed with PAH or CTEPH. Lung 2017; 195: 759-768.

2. Galiè N, Humbert M, Vachiery JL, Gibbs S, Lang I, Torbicki A, et al. $2015 \mathrm{ESC} / \mathrm{ERS}$ guidelines for the diagnosis and treatment of pulmonary hypertension: The Joint Task Force for the Diagnosis and Treatment of Pulmonary Hypertension of the European Society of Cardiology (ESC) and the European Respiratory Society (ERS): Endorsed by: Association for European Paediatric and Congenital Cardiology (AEPC), International Society for Heart and Lung Transplantation (ISHLT). Eur Heart $J$ 2016; 37: $67-119$.

3. Sato H, Ota H, Sugimura K, Aoki T, Tatebe S, Miura M, et al. Balloon pulmonary angioplasty improves biventricular functions and pulmonary flow in chronic thromboembolic pulmonary hypertension. Circ $J$ 2016; 80: 1470-1477.

4. Miwa H, Tanabe N, Jujo T, Kato F, Anazawa R, Yamamoto K, et al. Long-term outcome of chronic thromboembolic pulmonary hypertension at a single Japanese pulmonary endarterectomy center. Circ J 2018; 82: 1428-1436.

5. Darocha S, Pietura R, Pietrasik A, Norwa J, Dobosiewicz A, Piłka M, et al. Improvement in quality of life and hemodynamics in chronic thromboembolic pulmonary hypertension treated with balloon pulmonary angioplasty. Circ J 2017; 81: 552-557.

6. Egoshi S, Horie J, Anami K, Imaizumi Y, Ichimaru K, Naotsuka $\mathrm{H}$, et al. Are the lifestyle and social background of male patients with chronic obstructive pulmonary disease related to health- 
related quality of life? Jpn J Health Promot Phys Ther 2018; 8: $57-63$.

7. Umemura S, Arima H, Arima S, Asayama K, Dohi Y, Hirooka $Y$, et al. The Japanese Society of Hypertension guidelines for the management of hypertension (JSH 2019). Hypertens Res 2019; 9: $1235-1481$

8. Baumgartner H, Falk V, Bax JJ, De Bonis M, Hamm C, Holm PJ, et al. 2017 ESC/EACTS guidelines for the management of valvular heart disease. Eur Heart J 2017; 38: 2739-2791.

9. Gillett MJ. International Expert Committee report on the role of the A1c assay in the diagnosis of diabetes. Diabetes Care 2009; 32: $1327-1334$.

10. JCS Joint Working Group. Guidelines for the diagnosis, treatment and prevention of pulmonary thromboembolism and deep vein thrombosis (JCS 2009): Digest version. Circ J 2011; 75: $1258-1281$

11. The Report of an American Academy of Sleep Medicine Task Force. Sleep-related breathing disorders in adults: Recommendations for syndrome definition and measurement techniques in clinical research. Sleep 1999; 22: 667-689.

12. Yamagata Y, Ikeda S, Nakata T, Yonekura T, Koga S, Muroya $\mathrm{T}$, et al. Balloon pulmonary angioplasty is effective for treating peripheral-type chronic thromboembolic pulmonary hypertension in elderly patients. Geriatr Gerontol Int 2018; 18: 678-684.

13. Rabin R, Charro F. EQ-5D: A measure of health status from the EuroQol Group. Ann Med 2001; 33: 337-343.

14. Tsuchiya A, Ikeda S, Ikegami N, Nishimura S, Sakai I, Fukuda T, et al. Estimating an EQ-5D population value set: The case of Japan. Health Econ 2002; 11: 341 - 353.

15. Adamus-Leach HJ, Mama SK, O'Connor DP, Lee RE. Income differences in perceived neighborhood environment characteristics among African American women. Environ Health Insights 2012; 6: $33-40$.

16. Inoue S, Ohya Y, Odagiri Y, Odagiri Y, Takamiya T, Kamada $\mathrm{M}$, et al. Perceived neighborhood environment and walking for specific purposes among elderly Japanese. J Epidemiol 2011; 21: $481-490$

17. Lewczuk J, Piszko P, Jagas J, Porada A, Wójciak S, Sobkowicz $\mathrm{B}$, et al. Prognostic factors in medically treated patients with chronic pulmonary embolism. Chest 2001; 119: 818-823.

18. Sugimura K, Fukumoto Y, Satoh K, Nochioka K, Miura Y, Aoki T, et al. Percutaneous transluminal pulmonary angioplasty markedly improves pulmonary hemodynamics and long-term prognosis in patients with chronic thromboembolic pulmonary hypertension. Circ J 2012; 76: 485-488.

19. Hoole SP, Coghlan JG, Cannon JE, Taboada D, Toshner M, Sheares K, et al. Balloon pulmonary angioplasty for inoperable chronic thromboembolic pulmonary hypertension: The UK experience. Open Heart 2020; 7: e001144.

20. Minatsuki S, Kodera S, Kiyosue A, Yonekura T, Koga S, Muroya $\mathrm{T}$, et al. Balloon pulmonary angioplasty improves quality of life in Japanese patients with chronic thromboembolic pulmonary hypertension. J Cardiol 2020; 76: 205-210.

21. Yoshimi S, Tanabe N, Masuda M, Sakao S, Uruma T, Shimizu $\mathrm{H}$, et al. Survival and quality of life for patients with peripheral type chronic thromboembolic pulmonary hypertension. Circ $J$ 2008; 72: 958-965.

22. Urushibara T, Tanabe N, Suda R, Kato F, Kasai H, Takeuchi $\mathrm{T}$, et al. Effects of surgical and medical treatment on quality of life for patients with chronic thromboembolic pulmonary hypertension. Circ J 2015; 79: 2696-2702.

23. Fukui S, Ogo T, Takaki H, Ueda J, Tsuji A, Morita Y, et al. Efficacy of cardiac rehabilitation after balloon pulmonary angioplasty for chronic thromboembolic pulmonary hypertension. Heart 2016; 102: 1403-1409.

24. Thomassen R, Vandenbroucke JP, Rosendaal FR. Antipsychotic medication and venous thrombosis. Br J Psychiatry 2001; 179: $63-66$.

25. Hägg S, Spigset $\mathrm{O}$, Söderström TG. Association of venous thromboembolism and clozapine. Lancet 2000; 355: 1155-1156.

26. Jiang W, Kuchibhatla M, Clary GL, Cuffe MS, Christopher EJ, Alexander JD, et al. Relationship between depressive symptoms and long-term mortality in patients with heart failure. Am Heart $J$ 2007; 154: $102-108$.

27. Saarni SI, Viertiö S, Perälä J, Koskinen S Lönnqvist J, Suvisaari J. Quality of life of people with schizophrenia, bipolar disorder and other psychotic disorders. Br J Psychiatry 2010; 197: $386-394$.

28. Tajima H, Kasai H, Tanabe N, Sugiura T, Miwa H, Naito A, et al. Clinical characteristics and prognosis in patients with chronic thromboembolic pulmonary hypertension and a concomitant psychiatric disorder. Pulm Circ 2019; 9: 2045894019836420.

29. Menec VH. The relation between everyday activities and successful aging: A 6-year longitudinal study. J Gerontol B Psychol Sci Soc Sci 2003; 58: S74-S82.

30. Tanabe N, Sugiura T, Tatsumi K. Recent progress in the diagnosis and management of chronic thromboembolic pulmonary hypertension. Respir Investig 2013; 51: 134-146. 